\title{
Obiettivo: aumentare l'utilizzo della dialisi peritoneale in Italia
}

\author{
Giovambattista Virga
}

Unità Operativa Ospedaliera di Nefrologia e Dialisi, Ospedale di Camposampiero, Camposampiero (PD)

\begin{abstract}
OBJECTIVE: TO INCREASE THE USE OF PERITONEAL DIALYSIS IN ITALY
Abstract. Despite being less expensive than hemodialysis as dialysis technique, peritoneal dialysis (PD) in Italy is still used by only about $10 \%$ of dialysis patients. Important tools to increase its use could be not only to modify the cultural approach to it, but also to use PD in every Dialysis Unit and to provide a continue education for updating the nephrologists dealing with PD.
\end{abstract}

Key words: Peritoneal dialysis, Incidence, Prevalence

Conflict of interest: None.

Financial support: None.

Accettato: 2 Agosto 2013

La dialisi peritoneale (DP) è una tecnica dialitica non inferiore all'emodialisi in quanto a sopravvivenza dei pazienti ma di costo minore e, probabilmente, in grado di garantire un superiore benessere soggettivo.

L'utilizzo della DP in Italia è ancora ridotto a un valore di poco superiore al $10 \%$ dei pazienti dializzati (Registro Italiano di Dialisi e Trapianto, RIDT, 2009) mentre, in Paesi come Messico e Hong Kong, è superiore al 50\% (1).

L'uso della DP in Italia è anche molto variabile da regione a regione: dai dati del RIDT relativi al 2007, riguardanti 13/20 regioni, il $13.8 \%$ dei pazienti ha iniziato il trattamento sostitutivo con DP ma, mentre 3 regioni superavano la soglia del 20\% (Veneto, Liguria e Abruzzo), sette regioni non superavano il 10\% (Alto Adige, Trentino, Emilia Romagna, Lazio, Puglia, Calabria e Sardegna) e una regione era allo 0\% (Basilicata) (2). Erano immessi in DP in modo significativamente minore i pazienti anziani ( $>75$ anni), i diabetici e quelli con nefropatie vascolari, pielonefrite e familiari tipo ADPKD (2). Nei soli centri che utilizzano la DP, dai dati del Censimento del 2008, l'incidenza è risultata del 22.9\% (24.3\% nel 2005) e la prevalenza del 16.7\% (3). Dal 2005 al 2008, l'incidenza e la prevalenza sono diminuite e lo shift verso l'HD è peggiorato $(11.8 \% \rightarrow 12.4 \%)(3)$.

È ragionevole porsi come obiettivo in Italia un aumento della prevalenza della DP con il raggiungimento di una percentuale del 20\% sul territorio nazionale nei prossimi anni.

Per incrementare la prevalenza di pazienti in DP in Italia andrebbero aumentate l'incidenza e la sopravvivenza della tecnica e del paziente ("retention").
L'incidenza potrebbe aumentare:

1. modificando l'atteggiamento culturale verso la DP, a iniziare dalle Scuole di Specializzazione in Nefrologia, dove la DP dovrebbe essere presente come materia obbligatoria e con un programma condiviso anche dalla Società Italiana di Nefrologia (GdSDP), affinché alcune categorie di pazienti non debbano più essere considerate inidonee alla DP;

2. prevedendo una modalità di rimborso per le famiglie dei pazienti in tutte le regioni, sul modello della regione Piemonte, che aiuterebbe l'inserimento in DP di molti pazienti anziani o incapaci di eseguire da soli la DP;

3. incentivando tutte le Unità di Nefrologia e Dialisi a inserire un programma di DP;

4. aiutando i pazienti nella scelta della DP, spesso influenzata da informazioni fuorvianti ottenute da soggetti non accreditati, con mezzi audiovisivi o altri strumenti idonei allo scopo;

5. prevedendo la possibilità di addestramento per alcuni nefrologi e/o chirurghi al posizionamento del catetere peritoneale in centri designati.

La "retention" potrebbe aumentare:

1. con l'addestramento al posizionamento del catetere peritoneale;

2. con un programma di aggiornamento, anche on-line, per i nefrologi che si dedicano alla DP, per migliorare non solo l'outcome dei pazienti in DP, ma anche la sopravvivenza della tecnica, che potrebbe giovarsi di approcci più corretti relativi a tecniche e a schemi dialitici, di terapie per problemi specifici e di iter diagnostici aggiornati.

Quindi, per incrementare la prevalenza dei pazienti in DP in Italia, va modificato soprattutto l'atteggiamento culturale ver- 
so la DP, devono essere introdotte alcune nuove importanti norme e deve essere mantenuto e migliorato il programma di aggiornamento continuo accessibile ai nefrologi.

\section{Riassunto}

La dialisi peritoneale (DP) è una tecnica dialitica di costo minore rispetto all'emodialisi, ma ancora poco utilizzata in Italia (10\% circa dei dializzati). Per incrementare la sua prevalenza in Italia, andrebbero aumentate l'incidenza e la "retention". L'incidenza potrebbe migliorare inserendo la DP come materia nelle Scuole di Specializzazione in $\mathrm{Ne}$ frologia per modificare l'atteggiamento culturale, prevedendo una modalità di rimborso per le famiglie dei pazienti, inserendo in tutte le Unità di Dialisi un programma di DP, aiutando i pazienti nella scelta della DP con strumenti idonei allo scopo e prevedendo la possibilità di addestramento per alcuni nefrologi e/o chirurghi al posizionamento del catetere peritoneale. La "retention" può essere migliorata con l'addestramento al posizionamento del catetere peritoneale e creando un programma di aggiornamento continuo, anche on-line, per i Nefrologi che si dedicano alla DP.
Parole chiave: Dialisi peritoneale, Incidenza, Prevalenza

Dichiarazione di conflitto di interessi: L'Autore dichiara di non avere conflitto di interessi.

Contributi economici degli autori: L'Autore dichiara di non aver ricevuto sponsorizzazioni economiche per la preparazione dell'articolo.

Indirizzo degli Autori:

Dr. Giovambattista Virga

Unità Operativa Ospedaliera di Nefrologia e Dialisi

Ospedale di Camposampiero

Via P. Cosma 1

35012 Camposampiero (PD)

virgolino14@libero.it

\section{Bibliografia}

1. Jain AK, Blake P, Cordy P, Garg AX. Global trends in rates of peritoneal dialysis. J Am Soc Nephrol 2012; 23: 533-44.

2. Nordio M, Nichelatti M, Maggiore U, Limido A, Antonucci F. Valutazione della qualità delle cure mediante modelli gerarchici nel Registro Italiano di Dialisi e Trapianto. [Assessment of tre- atment quality by hierarchical models in the Italian dialysis and transplantation registry]. G Ital Nefrol 2011; 28 (2): 195-200.

3. Marinangeli G, Cabiddu G, Neri L, Viglino G, Russo R, Teatini U. Old and new perspectives on peritoneal dialysis in Italy emerging from the Peritoneal Dialysis Study Group Census. Perit Dial Int 2012; 32: 558-65. 